to investigate the influence of two different compression intensities throughout a 2 hour standing exposure on lower leg swelling, complaints, and wearing comfort.

Methods 40 healthy subjects participated in this randomised cross-over experiment with three 2 hour standing exposures that were tested on separate days. In condition A, subjects did not wear compression stockings; in condition B and C, subjects wore compression stockings medical class I (18$21 \mathrm{mmHg})$ and class II $(23-32 \mathrm{mmHg})$, respectively. Lower leg swelling was quantified by measures of lower leg volume (water plethysmography) and bioelectrical impedance before and after each standing exposure. Level of discomfort was assessed every $30 \mathrm{~min}$ (11-point Likert-Scale) and wearing comfort was measured at the end of the exposure using a custom-made standardised questionnaire. The study was approved by the ethics committee of the University of Tuebingen.

Result Preliminary results $(n=19)$ indicated that wearing compression stockings reduced lower leg swelling compared to wearing no stockings, but there was no difference in leg swelling between the two compression classes. Levels of discomfort were rather low and did not significantly differ across conditions. Higher levels of wearing comfort were found for the class I compared to the class II stockings.

Discussion These preliminary results suggest that in healthy subjects the lower compression intensity might be as effective as the higher intensity in reducing lower leg swelling. This is an important finding since compliance of wearing compression stockings increases with lower compression intensities, which is supported by the increased wearing comfort for the lower compression class in this study.

\section{POLITICS IN OCCUPATIONAL HEALTH: EXAMPLE OF AN EVIDENCE-BASED DRIVEN PROPOSAL FOR IMPROVING UPPER-EXTREMITY MUSCULOSKELETAL DISORDER COMPENSATION IN FRANCE}

1,2Alexis Descatha*. 'AP-HP, EMS (Samu92), Occupational Health Unit, University hospital of West suburb of Paris, Poincare site, F92380 Garches, France; ${ }^{2}$ Univ Versailles St-Quentin, F-78035, Versailles, France; Inserm, UMS 011 UMR1168, F-94807, Villejuif, France

\subsection{6/oemed-2018-ICOHabstracts.793}

Introduction In a context of heterogeneous practices, the French government set up a committee and this committe have mandated a technical working group for improving compensation for upper-extremity musculoskeletal disorders.

Methods After a state of the art based on international recommendations and French data to take into account the particular nature of compensation in France, a technical working group followed a Delphi-like process for suggesting a method of evaluation. A different group evaluated the work at the end with a validation process.

Result A systematic review has been performed, supplemented with opinions of International experts solicited individually. Analyses of available data were carried out on actual compensation and on a French cohort, as well as an inventory of the various French compensation methods. From this state of the art, the technical working group proposed a first version which was discussed in a workshop, allowing after two rounds a draft that a majority agreed upon $(>70 \%$ of total agreement). An independent group read and evaluated the work, with over 90\% total agreement, and allowed minor comments before the final report.

Discussion In one year, this project allowed the development of an evidence-based approach aimed at improving compensation for upper-extremity musculoskeletal disorders. An appropriation phase is necessary involving by end-users (i.e. insurance practitioners, with development of practical tools, clinical vignettes), and an economical evaluation.

\section{WORK EXPOSURES AND THEIR RELATIONSHIP TO THE DEVELOPMENT OF OSTEOARTHRITIS: A SYSTEMATIC REVIEW}

${ }^{1}$ Emma Irvin*, 'Monique Gignac, ${ }^{1}$ Kimberley Cullen, 'Dwayne Van Eerd, ${ }^{2}$ Catherine Backman, ${ }^{3}$ Dorcas Beaton, ${ }^{1}$ Siobhan Cardoso, ${ }^{1}$ Quenby Mahood, ${ }^{2}$ Christopher McLeod. ${ }^{1}$ Institute for Work and Health, Toronto, Canada; ${ }^{2}$ University of British Columbia, Vancouver, Canada; ${ }^{3}$ University of Toronto, Toronto, Canada

\subsection{6/oemed-2018-ICOHabstracts.794}

Introduction Osteoarthritis (OA) ranks among the top ten causes of disability worldwide. Of increasing interest are occupational factors that contribute to the development of $\mathrm{OA}$ or aggravate its trajectory. This systematic review synthesised evidence for the relationship between work exposures and the development of OA to inform work disability prevention messaging and decision making and to identify future knowledge needs.

Methods We implemented the systematic review process developed by the Institute for Work and Health and an adapted best evidence synthesis. Four electronic databases were searched from inception until May 2015. Articles that described the impact of work on OA were included in the review and examined knees, hips, spine, wrist, hands, or fingers, shoulder, ankle, foot, or toes, neck, elbow.

Result The search yielded 3379 non-duplicate references with 67 studies meeting our inclusion criteria. Study designs included cross-sectional surveys $(n=22)$, case-control studies $(n=22)$, prospective cohorts $(n=14)$, and retrospective cohorts $(n=9) .96 \%$ of studies measured OA using reliable and valid measures. Measurement of work exposures was more variable with $33 \% \quad(n=28)$ of studies not well described and $18 \%$ $(n=12)$ using instruments with questionable or unknown reliability and validity. There was strong or moderate evidence of an increased risk for developing OA in hips or knees for several occupational tasks (e.g., lifting/load bearing activities, full body vibration, kneeling/bending/squatting) and for not having an increased risk of OA for walking, sitting, driving, climbing. Dose response data were highly variable and could not be synthesised for recommendations.

Discussion This systematic review highlights that strong evidence exists for occupational risks and the development of $\mathrm{OA}$ in some joints, like knees and hips. However, data on complex job tasks (e.g., bending and lifting simultaneously) and dose response information are lacking. This is essential information going forward for prevention and early intervention efforts. 